Jpn. J. Human Genet. 26, 97-99, 1981

\title{
EVOLUTION OF GENES BY EPIMETHEAN HINDSIGHT
}

\author{
(The Japan Society of Human Genetics Award Lecture) \\ Susumu OHNo \\ Division of Biology, City of Hope Research Institute, \\ Duarte, California 91010, USA
}

In the Greek mythology, Zeus assigned the task of creating living creatures to the two Titan brothers; Epimetheus and Prometheus. Upon the completion of their task, Zeus punished not Epimetheus for his hindsight, but Prometheus for the impertinence of his foresight. Indeed, in this Darwinian world of evolution by natural selection, foresight is the forbidden fruit. Consider the following example: almost any colony of domesticated rabbits contains a good number of individuals whose occular pupils do not dilate in response to locally administered atropine. The above is due to the polymorphism of a certain esterase locus that confers an atropine esterase allele to certain individual rabbits as already known in 1943. Oryctolagus cuniculus in the wild lived in habitats rich in Belladonas and other atropine-containing nightshades which they no doubt consumed from time to time. The possesser of atropine esterase must have enjoyed a distinct selective advantage under the circumstance. What if Prometheus was the creator of the rabbit species? By foreseeing the rabbit's propensity to consume atropine-containing plants, he would have endowed every rabbit with the atropine esterase locus, thus, precluding the rabbit species from undergoing one adaptive evolutionary change noted above.

The mechanism guided by hindsight alone is extremely inefficient; each evolutionary success being preceeded by numerous failures. We then realize that as far as the highly evolved organisms are concerned, the preoccupation of natural selection has always been the conservation of hard earned successes rather than the advocation of further adaptive changes. Our recently acquired knowledge of DNA and polypeptide sequences has indeed revealed the conservation principle in that the functionally critical active site of the molecule seldom undergoes evolutionary changes, whereas species differences are very evident in trivial parts of the molecule. In the sense, one might say that evolution is an exercise in trivialities; e.g., compare histone IV with fibrinopeptide A. While histone IV of a mammal and a plant differ from each other only by two of the 110 amino acid residues, fibrinopeptide $A$ of the horse differs from that of the donkey by two deletions and two amino acid substitutions.

As previously pointed out by many, an organism has to pay a certain toll for the conservation of the functional integrity of each gene, and this toll is determined 
by the deleterious mutation rate of that locus. It follows then that there is a finite upper limit to the number of DNA base pairs in the genome that can be surveyed natural selection. In mammals, the total number of DNA base pairs placed under the vigorous surveillance by natural selection was estimated to be roughly $7 \times 10^{7}$. Inasmuch as the relatively uniform mammalian genome consists of $3.5 \times 10^{9} \mathrm{DNA}$ base pairs, $98 \%$ or so of the genomic DNA are to be regarded as freely drifting junks or selfish DNAs. One cosequence of the above is that even the so-called euchromatic region of each mammalian chromosome can be likened to a barren desert; genes (coding sequences) being found here and there in the manner of oases. The average distance between two neighbouring mammalian genes was calculated to $35 \mathrm{KBP}$ (kilobase pairs). Not surprisingly, even the most closely linked mammalian genes such as the pair for human hemoglobin $\gamma^{\text {ala }}$ and $\gamma^{g l y}$ are found be separated by the distance of $3 \mathrm{KBP}$, and that the spacer length between obvious duplicates can approach the estimated average distance of $35 \mathrm{KBP}$; e.g., the 21 KBP-long spacer separates the immunoglobulin heavy chain constant region gene $\mathrm{C}_{\gamma 1}$ from $\mathrm{C}_{\gamma 2 b}$.

Being junks, spacer DNA sequences should be ignored by natural selection, thus, accumulating with impunity any and all randomly sustained mutational changes, and herein is found the ultimate paradox; for even junk spacers can sustain clearly deleterious mutations in the form of the inadvertent acquisition of promotor sites for RNA polymerase which would permit the transcription of junks as well as in the form of the inadvertent acquisition of coding sequences that may specify nonsense polypeptides which may even be toxic.

The presence of so-called intermediately repetitious sequences between the genes has long been known. In view of the above, this old fact acquires the new significance. As originally pointed out by Ed Southern, all repetitious sequences follow the predictable evolutionary fate: Each family of repeats arises as exact replica of the short, specific base sequence. Soon, these repeats disguise themselves as socalled intermediately repetitious repeats that are rather unfaithful copies of the sequence considerably longer than the original. At the final stage of degeneracy due to randomly sustained mutations, the family join the rank of senseless unique sequences.

What if each spacer sequence originated as the exact replica of the short sequence of specific quality such as the 20BP-long sequence: (AGCTG)(AGCTG) (AGCTG)(GGGTG)? Even after considerable degeneracy due to randomly sustained mutations, the sequence derived from the above would not readily yield either promotor sites or coding sequences. This is because the putative promotor sequence TATAATA known as the Hogness box contains no $\mathrm{GC}$, whereas in the above 20BP-long sequence, GC outnumbers $\mathrm{AT}$ by 13 to 7 . Furthermore, the above sequence when transcribed gives rise to three chain terminating UGA codons; one for each reading frame.

The spacer in front of the immunoglobulin heavy chain constant region $\mathrm{C}_{\mu}$ 
gene sequenced by Sakano et al. indeed has arisen as exact replica of the above noted 20BP-long sequence, for it still contained the five exact replica of (AGCTG) (AGCTG)(AGCTG)(GGGTG) in its midst, and this core was flanked on both sides by a few unfaithful copies of the 49 BP-long degenerate sequence: (AGCTC) c (AGGGG)(AGCCA) GGAC (AGGTG) GA (AG-TG)(TGGTG) GACCC AGGCA GAGC.

Expected extreme instability of spacer sequences as junks was also revealed by the spacer in front of the immunoglobulin heavy chain constant region $\mathrm{Ca}$ gene, for its particular region independently sequenced by Kataoka et al. and Davis et al. differed from each other by 20 or so base substitutions and about 65 deletions, in spite of the fact that both were derived from the same inbred mouse strain BALB/c. 